\title{
Consensus Development of a Core Content for a Standardized Medical Toxicology Curriculum for Medical Students
}

\author{
Charlotte Goldfine ${ }^{1}\left[\right.$ - Derrick Lung ${ }^{2} \cdot$ Gillian Beauchamp ${ }^{3} \cdot$ Ayrn O'Connor $^{4} \cdot$ Andrew Stolbach $^{5} \cdot$ Louise Kao $^{6}$. \\ Bryan Judge $^{7} \cdot$ Paul Wax $^{8} \cdot$ Rahul Patwari $^{9} \cdot$ Ziad Kazzi $^{10}$
}

Received: 27 August 2021 / Revised: 13 December 2021 / Accepted: 16 December 2021 / Published online: 28 January 2022

(c) American College of Medical Toxicology 2022

\begin{abstract}
Background Currently, no standardized core content in medical toxicology exists for medical students. The goals of this study were to (1) assess the current state and needs of medical toxicology clerkships and (2) develop a consensus-derived list of core topics that should be covered during a medical toxicology clerkship.

Methods We assembled a task force established by the American College of Medical Toxicology (ACMT) of nine experts in medical toxicology or emergency medicine. We developed a needs assessment survey that was sent to all medical student clerkship directors in medical toxicology. Based on their responses, we used a modified Delphi process to develop a consensus of core topics that should be covered during a medical student clerkship.

Results Nineteen out of 42 (45\%) clerkship directors completed the survey; 18 met inclusion criteria. The majority of clerkships were 4 weeks in duration with an average of 15 students/year. The three most common teaching methods used were bedside teaching $(n=17 / 18)$, classroom teaching $(n=17 / 18)$, and journal club $(n=14 / 18)$. All the clerkship directors $(n=18 / 18)$ reported they would use a standardized curriculum as well as educational content developed by ACMT. There was overwhelming consensus on the core topics which included, but were not limited to, pharmacology/toxicology; drugs; drugs of abuse; natural products; pharmacological basis of antidote use; toxicologic syndromes; vital sign abnormalities; initial management; supportive and other care; withdrawal syndrome management; industrial, household, and environmental toxins; differential diagnosis by clinical findings; and ABCs-resuscitation.

Conclusion The ACMT task force developed a medical toxicology clerkship core content. The task force also identified a need for shared resources among clerkships.
\end{abstract}

Keywords Medical education · Core content $\cdot$ Medical toxicology

Supervising Editor: Mark B. Mycyk, MD.

Charlotte Goldfine

cgoldfine@bwh.harvard.edu

1 Division of Medical Toxicology, Department of Emergency Medicine, Brigham and Women's Hospital, Boston, MA, USA

2 Division of Clinical Pharmacology and Medical Toxicology, Department of Emergency Medicine, San Mateo Medical Center, San Mateo, CA, USA

3 Department of Emergency and Hospital Medicine, Division of Medical Toxicology, Lehigh Valley Health Network, University of South Florida Morsani College of Medicine, Allentown, PA, USA

4 Banner - University Medical Center Phoenix, Phoenix, AZ, USA
5 Department of Emergency Medicine, Johns Hopkins University, Baltimore, MD, USA

6 Department of Emergency Medicine, Division of Medical Toxicology, Indiana University School of Medicine, Indianapolis, IN, USA

7 Department of Emergency Medicine, Michigan State University College of Human Medicine, Grand Rapids, MI, USA

8 Southwestern School of Medicine, University of Texas, Dallas, TX, USA

9 Rush Medical College, Chicago, IL, USA

10 Georgia Poison Center, Emory University, Atlanta, GA, USA 


\section{Introduction}

Poisoning is the leading cause of unintentional injuryrelated death [1,2]. In 2019, the nationwide death rate in the USA due to unintentional poisonings was 20 deaths per 100,000 , and over 2.1 million calls were made to US poison control centers for human poison exposures [3, 4]. Between the years of 2010-2018, a total of 66,248 cases were reported to a national surveillance registry, the Toxicology Investigators Consortium (ToxIC), representing medical toxicology consults voluntarily reported across 73 participating facilities [5].

Despite the importance of treating poisoning and overdose, there is no standardized medical education in toxicology. Some medical toxicology concepts may be taught in non-clinical courses (e.g., pharmacology), and students may get clinical exposure to toxicologic pathology within other clerkships. Medical students may receive limited exposure to the specialty during emergency medicine or pediatric emergency medicine clerkships, or are not formally exposed to medical toxicology at all. Furthermore, dedicated medical toxicology clerkships are not available at every institution.

Given the impact of toxicologic exposures, it is important for medical students to be educated on how to care for patients after such exposures. At the time of this study, there were 42 medical toxicology clerkships at US allopathic medical schools (total number of schools in 2017 was 147) [6]. However, the educational characteristics of these clerkships is not standardized and widely variable. Clerkships have different curricula and use different teaching methods. The aims of this study were to (1) assess the current state and needs of medical toxicology clerkships and (2) develop a consensus-based list of core topics that should be included in future curricula dedicated for medical toxicology clerkships in medical school.

\section{Methods}

The American College of Medical Toxicology's (ACMT) Education Committee and Clerkship Council for Medical Toxicology (CCMT) formed a task force with the goal of developing a medical student core content in medical toxicology that can be used for clerkship rotations. The CCMT is an ACMT committee composed of medical toxicology clerkship directors and medical student educators. We assembled a task force of experts in medical toxicology and emergency medicine. We invited prospective experts by email from a convenience sample consisting of members of CCMT and the professional network of medical toxicology and emergency medicine educators. Prospective experts were given a brief description of the project, objectives, expected timeline, and expected time commitment. Prospective experts were given the opportunity to decline participation and to name other potential experts that could substitute. All nine individuals invited to serve as expert members of the task force accepted. Each expert was board-certified in medical toxicology $(n=8 / 9)$ or emergency medicine $(n=9 / 9)$, and most had served or currently serve as a clerkship director at a medical school $(n=6 / 9)$. The majority of experts had between 14 and 20 years of experience as medical educators $(n=7 / 9)$. The other two experts had 6-7 years of experience as medical educators. The majority of experts who had served as clerkship directors had between 8 and 15 years of experience in that role $(n=4 / 6)$. One expert was clerkship director for 18 years, and one was clerkship director for 2 years. The task force chairs reviewed and summarized prior published literature regarding the core content of medical toxicology for emergency medicine residencies and clerkships, the core content for pediatric emergency medicine rotations, and the core content for medical toxicology fellowships [7-10].

To assess the current state of medical toxicology clerkship education, we sent an anonymous, 16-question electronic, multiple-choice, and free-text response survey to all the clerkship directors listed in the ACMT database for medical student clerkships in medical toxicology (Appendix). The survey was developed by two of the task force members with expertise in medical toxicology, emergency medicine, and medical education and was finalized by consensus of the task force. The survey was sent by email to the clerkship directors in July 2017 with two additional reminder emails 1.5 and 2.5 weeks after the initial email to complete the survey. The survey was open for 5 weeks from July to August 2017. The questions were designed to assess the current state and needs of the clerkships. The respondents were also given a list of 43 medical toxicology topic areas representing the core content of medical toxicology [7]. From that list, they were asked to select topic areas that should be included in a medical toxicology clerkship for medical students. Respondents were offered the opportunity to add additional topics that were not included in the list. The survey also asked clerkship directors to share their curriculum if available.

The task force met from May 2017 to June 2018 and utilized the initial steps for the Kern model (problem identification and needs assessment) for the development of the core content [11]. First, the task force identified a lack of core content and standardized toxicology education for medical students. The task force then used the survey responses from the clerkship directors as a targeted needs assessment. At subsequent meetings, the task force reviewed the core content for medical toxicology fellowship training and the 
results of the survey completed by the clerkship directors. We asked the expert panel to identify the desired length and format of the core content. After tabulating the results, one of the task force chairs moderated the discussion and followed a modified Delphi process to reach consensus among the group about the proposed clerkship core list of topics including assigning them into two categories: tier 1 (must be covered in the clerkship) and tier 2 (could be covered). Topics that received greater than $75 \%$ agreement among experts were accepted as tier 1 topics. Those topics that received $50-75 \%$ of agreement were accepted as tier 2 topics. When greater than $75 \%$ of experts disagreed with preliminary topics, those topics were considered rejected and thus discarded. The process was continued until a final number of core content topics was reached. The final list of core topics was then presented to the expert panel for approval.

\section{Results}

The survey was completed by 19 out of 42 programs listed in the ACMT Clerkship Directory (45\% response rate). One respondent no longer had a clerkship at their site so was excluded from the analysis. The majority of the clerkships were 4 weeks in duration $(n=14 / 18)$. The remainder of the clerkships offer a choice of a 2- or 4-week rotation $(n=4 / 18)$. The mean number of students rotating through the clerkships of responding programs per year was 15 (range 1-40 students per year).

Most of the clerkship directors $(n=16 / 18)$ reported having a toxicology curriculum with goals, objectives, or a list of important topics to be covered. The three most common teaching methods used included bedside teaching $(n=17 / 18)$, classroom teaching $(n=17 / 18)$, and journal club $(n=14 / 18)$. The least commonly used teaching methods were asynchronous web-based instruction $(n=9 / 18)$, synchronous web-based modalities such as live webinars $(n=5 / 18)$, and simulation $(n=3 / 18)$. The clerkship directors expressed interest in increasing use of these educational tools as well as assembling a compendium of landmark articles from the toxicology literature.

The majority of medical students at the surveyed sites are also taught medical toxicology outside of a dedicated clerkship $(n=13 / 18)$, during emergency medicine or pediatric clerkships. Most of the teaching at these institutions is provided by medical toxicologists $(n=16 / 18)$, fellows $(n=11 / 18)$, or clinical pharmacologists who have undergone clinical toxicology fellowship training $(n=9 / 18)$. Clerkship students are evaluated using end-of-clerkship presentations $(n=14 / 18)$, written evaluations $(n=12 / 18)$, and pre- and post-clerkship tests $(n=8 / 18)$.

Four curricula were shared with the task force. All four included clearly defined goals and objectives for the students. Some examples of these goals include "To acquaint the student with the pathophysiology, diagnosis and treatment of acute and chronic poisonings by pharmaceuticals, illicit substances, household products, industrial chemicals and natural toxins;" "Develop clinical skills to assess a poisoning emergency"; "Learn how to access, critically appraise, and use the findings of the medical literature in clinical situations"; and "Be familiar with the operation of the Regional Drug and Poison Information Center and its role in the prevention and management of poisonings." Three of the shared curricula were designed to be covered in 4 weeks. Additionally, two of the curricula included assigned readings and two included cases or questions designed for the student to complete during their clerkship.

All of the respondents $(n=18)$ reported that they would use a standardized curriculum for a medical toxicology clerkship developed by ACMT. Sixteen of the clerkship directors responded about the topics that should be included in the core curriculum. After the modified Delphi process, the core topics selected by the task force are listed in Table 1 .

\section{Discussion}

Performing a needs assessment survey was an important step to better understand the current status of medical toxicology education for medical students. The goal of creating a core content is to provide additional support for existing clerkships and to provide a framework and resources that can be used to create new clerkships at other institutions. Exposing medical students to medical toxicology education is important both for the growth of the specialty, and more importantly, to improve the care of patients presenting with a toxicological illness.

The ACMT is a non-profit, membership organization whose mission is "advancing the toxicologic care of patients and populations; and advocating for the specialty of medical toxicology [12]." ACMT has an Education Committee and a Clerkship Council for Medical Toxicology dedicated to increasing awareness about the field and specialty among medical students and to providing support for educators and clerkship directors. ACMT and its committees provide a readily available resource to develop the core curriculum content and further educational opportunities for medical students in medical toxicology.

This core content list is meant to serve as an outline of topics that are necessary for medical student education. As the majority of the clerkships surveyed are 4 weeks in duration, the core content is intended to be covered during a dedicated toxicology clerkship in that period of time. However, the tier 1 topics could be covered in a 2-week rotation. The tiered core content allows clerkship directors to tailor the format as needed for length of time or different levels of 
Table 1 Proposed medical toxicology clerkship core content

\begin{tabular}{|c|c|}
\hline Tier 1 & $\begin{array}{l}\text { 1.1 Pharmacology/toxicology } \\
\text { 1.2 Drugs } \\
\text { 1.3 Drugs of abuse } \\
\text { 1.4 Natural products } \\
\text { 1.5 Pharmacological basis of antidote use } \\
\text { 1.6 Toxicologic syndromes } \\
\text { 1.7 Vital sign abnormalities } \\
\text { 1.8 Initial management } \\
\text { 1.9 Supportive and other care } \\
\text { 1.10 Withdrawal syndrome management } \\
\text { 1.11 Industrial, household, and environmental toxins } \\
\text { 1.12 Differential diagnosis by clinical findings } \\
\text { 1.13 ABCs-resuscitation }\end{array}$ \\
\hline Tier 2 & $\begin{array}{l}\text { 2.1 Poison centers } \\
\text { 2.2 Laboratory and other diagnostic assessments } \\
\text { 2.3 Mechanisms of reproductive and developmental toxicity } \\
\text { 2.4 Pediatric and reproductive syndromes (developmental disorders, teratogenesis) } \\
\text { 2.5 Radiation syndromes } \\
\text { 2.6 Toxic outbreaks of historical significance (e.g., Yusho, toxic oil) } \\
\text { 2.7 Response to hazardous materials (Hazmat) incidents, including terrorism } \\
\text { 2.8 Warfare and terrorism } \\
\text { 2.9 Molecular components/mechanisms } \\
\text { 2.10 Assay methods and interpretation }\end{array}$ \\
\hline
\end{tabular}

learners. The current structure allows flexibility in implementation as some topics are broader, while other narrow topics may be able to be combined and covered in a single session or lecture and in the style preferred by the educator.

There are several limitations of this study. First, the core content was created as a consensus from a task force of medical toxicologists, emergency physicians, and clerkship directors. A consensus can vary depending on the size of the group and the opinions of the participating members. The consensus-derived process created a broad core content for a 2- or 4-week clerkship. While all of these topics were determined to be important, it may be difficult to cover every aspect of these topics in 4 weeks. Future work will focus on asking other key stakeholders in undergraduate medical education to determine which topics should be covered in a medical toxicology clerkship and which to integrate into existing medical school curricula.

The survey itself also has some limitations as it was also developed by consensus and was not piloted. This may have led to questions not being interpreted uniformly among participants. For example, the question "Do you currently have a medical toxicology curriculum with goals, objectives, and/ or topics?" could be interpreted in different ways. The survey was only completed by $45 \%$ of the current clerkship directors; therefore, the results may not be representative of the needs of all of the programs. It is also important to acknowledge the majority of participants $(n=16 / 18)$ are members of ACMT and therefore may be biased to use resources developed by the organization. The survey respondents also included some members of the task force, and one of the four shared curricula was provided by a task force member. Finally, this study was conducted before the COVID-19 pandemic and the widespread incorporation of the virtual format into undergraduate medical education. In order to better understand the applicability of the core content, next steps will be to disseminate and implement the core content and obtain feedback from the clerkship directors.

The ultimate goal of this project is to create a standardized medical toxicology curriculum. As there was interest in educational content created by ACMT, future steps will focus on creating resources that can be used to implement the core content. Specific objectives will be to develop and promote the resources that the clerkship director survey identified as currently lacking. This includes compiling a list of landmark articles from the toxicology literature, creating simulation case content, and increasing asynchronous learning opportunities geared towards medical students.

\section{Conclusions}

The ACMT task force has developed a core content for medical student training in medical toxicology. The core content can be used by clerkship directors and the ACMT as a guide to standardize medical toxicology education for medical students. Additionally, there is a need for shared resources among clerkships.

\section{Appendix. Needs Assessment Questions}

School of medicine/hospital:

Clerkship director name:

Duration of clerkship: 
- 1 week

- 2 weeks

- 3 weeks

- 4 weeks

- A month

- Other (please specify)

Approx. how many students in program per year:

Do you currently have a medical toxicology curriculum with goals, objectives, and/or topics?

- Yes

- No

If yes, would you be willing to share with the task force your curriculum, such as clerkship goals, objectives, list of topics, suggested readings, or other curricular elements? If so, we will contact you separately by email. The information you provide to us will be used for internal purposes only. It will not be made public or shared with outside organizations.

- Yes

- No

What methods do you currently use for teaching during your clerkship? Select all that apply.

- Web-based instruction-synchronous (e.g., live webinars)

- Web-based instruction-asynchronous, on-demand (enduring material such as podcasts or modules)

- Bedside teaching

- Classroom-based (roundtable, formal lecture, etc.)

- Simulation

- Journal club

- Compendium of toxicology literature (reading list, for example)

- Grand rounds

- Other (please specify)

What methods would you like to use for teaching during your clerkship that you don't currently use? Select all that apply.

- Web-based instruction—synchronous (e.g., live webinars)

- Web-based instruction-asynchronous, on-demand (enduring material such as podcasts or modules)

- Bedside teaching

- Classroom-based (roundtable, formal lecture, etc.)

- Simulation

- Journal club
- Compendium of toxicology literature (reading list, for example)

- Grand rounds

- Other (please specify)

Is medical toxicology content being taught outside of your medical toxicology clerkship (i.e., emergency medicine clerkship or pediatric clerkship)?

- Yes

- No

Who teaches toxicology content to medical students at your institution (either on tox clerkship or within other clerkships such as EM or pediatrics)? Select all that apply.

- Fellows

- Residents

- Attending emergency medicine physician (w/out medical toxicology training)

- Clinical pharmacists/clinical toxicologists

- Medical toxicologists

- Other (please specify)

How do you evaluate your clerkship students? Select all that apply.

- Pre-test/post-test

- Regular quizzes

- End-of-clerkship presentation

- Written evaluation

- Verbal evaluation

- Do not evaluate

- Other (please specify)

Would you use a standardized curriculum for a medical toxicology clerkship developed by ACMT?

- Yes

- No

Would you like ACMT to develop educational content for a standardized medical toxicology curriculum?

- Yes

- No

If yes, would you be willing to help develop educational content for an ACMT standardized medical toxicology curriculum?

- Yes

- No 
What content do you think should be included in a medical toxicology clerkship for medical students? Look at the list of medical toxicology core content categories below and select all that apply:

1.1 Pharmacology/toxicology

1.2 Molecular components/mechanisms

1.3 Cytotoxic mechanisms (e.g., apoptosis, microtubular dysfunction)

1.4 Principles of radiation (e.g., decay, units)

1.5 Mutagenesis and carcinogenesis

1.6 Mechanisms of reproductive and developmental toxicity

2.1 Drugs (pharmaceuticals)

2.2 Drugs of abuse

2.3 Industrial, household, and environmental toxicants

2.4 Natural products (e.g., plants, envenomations)

2.5 Warfare and terrorism

2.6 Radiological

3.1 Toxicologic syndromes (e.g., cholinergic, opioid)

3.2 Vital sign abnormalities/syndromes

3.3 Differential diagnosis by clinical finding

3.4 Pediatric and reproductive syndromes (developmental disorders, teratogenesis)

3.5 Radiation syndromes

3.6 Syndromes attributed to the environment, not specified elsewhere (e.g., mold, multiple chemical sensitivity)

3.7 Toxic outbreaks of historical significance (e.g., Yusho, toxic oil)

4.1 ABCs-resuscitation

4.2 Initial management

4.3 Pharmacological basis of antidote use

4.4 Supportive and other care

4.5 Withdrawal syndrome management

4.6 Radiation exposure management (e.g., triage, medical therapy)

5.1 Criteria for causal inference (e.g., biological plausibility, consistency,

dose-response relationship, strength of association, temporal relationship)

5.2 Monitoring

5.3 Occupational assessment and prevention

5.4 Principles of epidemiology and study design

5.5 Risk assessment and management

5.6 Poison centers

5.7 Response to hazardous materials (Hazmat) incidents, including terrorism

5.8 Role of federal and international agencies in toxicology

5.9 Injury prevention

5.10 Medical publishing

5.11 Consultation resources (e.g., databases, National Library of Medicine) 6.1 Assay methods and interpretation

6.2 Detection and interpretation of performance enhancing substances

6.3 Laboratory and other diagnostic assessments

6.4 Forensics

6.5 Legal drugs of abuse (e.g., psychomotor impairment)

6.6 Legal ethanol (e.g., legal aspects of alcohol use)

6.7 Medical legal issues

Other (please specify)
Acknowledgements Special thanks to the clerkship directors who participated in this study and the ACMT Clerkship Council and Education Committee.

Sources of Funding

None

\section{Declarations}

Conflicts of Interest PW is the executive director of ACMT. LK, AS, JK, $\mathrm{BJ}$, and $\mathrm{AO}$ are on the board of directors of ACMT.

\section{References}

1. Hedegaard H, Miniño A, Warner M. Drug overdose deaths in the United States, 1999-2018. NCHS Data Brief. 2020;356:1-8.

2. Desai NM, Mistry RD, Brou L, Boehnke ME, Lee JS, Wang GS. Pediatric exposures reported to the Toxicology Investigators Consortium, 2010-2015. Pediatr Emerg Care. 2021; 37(12): e1039-e1043 https://doi.org/10.1097/pec.0000000000001893.

3. Centers for Disease Control and Prevention NCfHS: underlying cause of death 1999-2019. https://wonder.cdc.gov/controller/datar equest/D76;jsessionid=471241C4C3C8FF64A8A2F11BDE3D\# Citation (2020). Accessed October 202021.

4. Gummin DD, Mowry JB, Beuhler MC, Spyker DA, Brooks DE, Dibert KW, et al. 2019 annual report of the American Association of Poison Control Centers' National Poison Data System (NPDS): 37th annual report. Clin Toxicol. 2020;58(12):1360-541. https://doi. org/10.1080/15563650.2020.1834219.

5. Spyres MB, Farrugia LA, Kang AM, Calello DP, Campleman SL, Pizon A, et al. The Toxicology Investigators Consortium case registry-the 2018 annual report. J Med Toxicol. 2019;15(4):228-54. https://doi.org/10.1007/s13181-019-00736-9.

6. Barzansky B, Etzel SI. Medical schools in the United States, 20172018. JAMA. 2018;320(10):1042-50.

7. Nelson LS, Baker BA, Osterhoudt KC, Snook CP, Keehbauch JN. The 2012 core content of medical toxicology. J Med Toxicol. 2012;8(2):183-91. https://doi.org/10.1007/s13181-012-0223-5.

8. Tews MC, Wyte CMD, Coltman M, Grekin PA, Hiller K, Oyama LC, et al. Developing a third-year emergency medicine medical student curriculum: a syllabus of content. Acad Emerg Med. 2011;18:S36-40.

9. Manthey DE, Ander DS, Gordon DC, Morrissey T, Sherman SC, Smith MD, et al. Emergency medicine clerkship curriculum: an update and revision. Acad Emerg Med. 2010;17(6):638-43.

10. Askew KL, Weiner D, Murphy C, Duong M, Fox J, Fox S, et al. Consensus development of a pediatric emergency medicine clerkship curriculum. West J Emerg Med. 2014;15(6):647-51. https:// doi.org/10.5811/westjem.2014.4.20007.

11. Thomas PA, Kern DE, Hughes MT, Chen BY. Curriculum development for medical education: a six-step approach. JHU Press; 2016.

12. Toxicology ACoM: ACMT Facts. https://www.acmt.net/ACMT_ Fact_Sheet.html. Accessed 26 May 2021.

Publisher's Note Springer Nature remains neutral with regard to jurisdictional claims in published maps and institutional affiliations. 